\title{
Lipocalin-2 Alleviates LPS-Induced Inflammation Through Alteration of Macrophage Properties
}

\author{
Huahua $\mathrm{Du}{ }^{\prime}$ \\ Li Liang' \\ Jiahui $\mathrm{Li}^{\prime}$ \\ Qingqing Xiong' \\ $\mathrm{Xin} \mathrm{Yu}^{2}$ \\ Hong $\mathrm{Yu}^{3}$
}

'MoE Key Laboratory of Molecular Animal Nutrition, College of Animal Science, Zhejiang University, Zhejiang, 3 I0058, People's Republic of China; ${ }^{2}$ Department of Anesthesia, Sir Run Run Shaw Hospital, School of Medicine, Zhejiang University, Zhejiang, 310016, People's Republic of China; ${ }^{3}$ Department of General Surgery, Sir Run Run Shaw Hospital, School of Medicine, Zhejiang University, Zhejiang, 310016, People's Republic of China
Correspondence: Hong Yu

Department of General Surgery, Sir Run Run Shaw Hospital, School of Medicine,

Zhejiang University, Zhejiang, 310016,

People's Republic of China

Tel +86-57I-860448I7

Fax +86-57I-86090063

Email blueyu000@zju.edu.cn
Purpose: Lipocalin-2 (Lcn2) is an acute-phase protein and elevated in several inflammatory diseases. This study aimed to determine whether Lcn2 alleviates inflammation and explore the underlying cellular mechanisms.

Methods: $\mathrm{C} 57 \mathrm{BL} / 6 \mathrm{Lcn} 2$-deficient $\left(\mathrm{Lcn} 2^{-/}\right)$male mice were intraperitoneally injected with lipopolysaccharide (LPS) to build systemic inflammation model. The inflammatory processes were investigated. The morphology of villi was measured by scanning electron microscopy (SEM). The levels of inflammatory factors were detected by ELISA and qPCR analysis. The production of Len2 was determined with immunofluorescence staining by confocal microscope. The molecular mechanism of Len2 in bone marrow-derived macrophages (BMDMs) was analyzed by mass spectrometry (MS)-based quantitative proteomic analysis.

Results: Compared to wild-type (WT) mice injected with LPS, Lcn $2^{-/}$mice injected with LPS showed increased inflammatory damage in jejunum and ileum, and significantly elevated the levels of multiple pro-inflammatory cytokines. After determining that Lcn2 was mainly located in the cytoplasm of macrophages, we isolated BMDMs from $\mathrm{Lcn} 2^{-/-}$mice to evaluate their function. During LPS challenge, transcripts of pro-inflammatory cytokines were all significantly increased in BMDMs from $\mathrm{Lcn} 2^{-/}$mice, while those of anti-inflammatory cytokines were significantly decreased when compared with the cytokines in BMDMs from WT mice. A label-free relative quantitation proteomics analysis showed that LPS-treated BMDMs from $\mathrm{Lcn} 2^{-/}$mice had elevated levels of pro-inflammatory pathways, but reduced phagocytosis and autophagy when compared with LPS-treated BMDMs from WT mice.

Conclusion: These findings demonstrated that $\operatorname{Lcn} 2$ was a potent protective factor in response to systemic inflammation and might be an indispensable factor for macrophage functions.

Keywords: Lcn2, systemic inflammation, macrophages, BMDMs

\section{Introduction}

Inflammation is an essential innate immune response, which is necessary for selfprotection against harmful irritants and pathogens. It is a localized physical condition, in which certain parts of the body become swollen, hot and often painful, especially in response to injuries and infections. Notably, surplus inflammation is deleterious and leads to multiple chronic diseases such as cancer, ${ }^{1}$ obesity, ${ }^{2}$ and coronary artery disease, ${ }^{3}$ which are all closely associated with altered immune homeostasis. Generally, the immune system has a well-established contribution to pro-inflammatory initiation and tissue wound healing. However, in many cases such as sepsis, over-activation of immune responses with sufficiently high levels of inflammatory cytokine secretion may cause severe tissue damage. ${ }^{4}$ 
Enhanced tissue infiltration of immune cells is one of the main histological features of inflammation. Macrophages are immune cells involved in both sterile and infectious inflammatory syndromes. ${ }^{5}$ Macrophages have been not only considered to be one of crucial mediators that trigger the inflammatory response by secreting various pro-inflammatory cytokines, but also involved in tissue repair and regeneration by secreting antiinflammatory cytokines and phagocytosis. ${ }^{6}$ However, the factors which trigger and balance the different function of macrophages during inflammation remain largely unknown. Thus, the question is which contents of the immune system can directly contribute to the process for the resolution of excessive immune responses.

Lipocalin-2 (Lcn2), also known as neutrophil gelatinase-associated lipocalin, siderocalin, or $24 \mathrm{p} 3$, is a $25 \mathrm{kDa}$ secretory glycoprotein, which is originally isolated from neutrophil granules. ${ }^{7}$ As a member of broader lipocalin family, Lcn2 forms a barrel-shaped tertiary structure with hydrophobic cavities that bind to a variety of lipophilic ligands, including prostaglandin, progesterone, retinoic acid, and fatty acids. ${ }^{8}$ Len 2 has been shown to play important roles in various biological processes, including iron delivery, ${ }^{9}$ apoptosis, ${ }^{10}$ kidney development, ${ }^{11}$ and innate immunity. ${ }^{12}$ Although mainly secreted by neutrophils, Lcn2 is also expressed in other cell types, such as hepatocytes, intestinal epithelia, and macrophages. ${ }^{13,14}$ Lcn2 has been reported to be an acute-phase protein with elevated levels in urine, serum, feces, and epithelium of patients with active inflammatory disease. ${ }^{15,16}$ However, the cellular mechanism whereby Lcn2 alleviates inflammation remains to be elucidated.

Therefore, in this study, we investigated the role of Lcn2 in inflammation processes using gene-targeted Lcn2-deficient $\left(\mathrm{Lcn}^{-/-}\right)$mice. We showed that, in response to inflammatory stimuli lipopolysaccharides (LPS), Lcn $2^{-/-}$mice exhibited more severe inflammation injury, in keeping with the dramatically increased levels of pro-inflammatory cytokines. Moreover, we found that Lcn2 was predominantly expressed in the cytoplasm of macrophages after LPS challenge. Functionally, proteomic results showed that bone marrow-derived macrophages (BMDMs) derived from $\mathrm{Lcn}^{-/-}$mice were defective in phagocytosis and autophagic degradation of surplus inflammatory determinants, thereby promoting pro-inflammatory responses.

\section{Materials and Methods}

\section{Animal Experiments}

C57BL/6 WT male mice (6 8 week) were obtained from Animal Center of Chinese Academy of Sciences (Shanghai, China) and C57BL/6 Lcn2-deficient $\left(\operatorname{Lcn} 2^{-/}\right)$ male mice (6 8 week) were got from Jackson Laboratory (USA), respectively. All mice were received food and water ad libitum, and housed under standard laboratory conditions on $12 \mathrm{~h}$ light-dark cycles in a specific pathogen-free environment. For systemic inflammation studies, $\mathrm{Lcn} 2^{-/}$and wild type (WT) mice were intraperitoneally injected with $2 \mu \mathrm{g} / \mathrm{g}$ body weight LPS (Sigma Aldrich, USA). A total of 48 mice were randomly assigned to be injected with LPS or saline ( $n=24$ each group), and euthanized by cervical dislocation at $0,8,16,24,32$, and $48 \mathrm{~h}$ after LPS injection ( $n=4$ per time point). Peripheral blood was obtained from the retro-orbital sinus for serum collection. Liver, kidney, spleen, duodenum, jejunum, ileum, and colon were collected for measurements of inflammatory injury and Lcn 2 expression. All mouse experiments were approved by the Animal Care Committee of Zhejiang University (ZJU2018-12141), and were performed in accordance with the animal ethics guidelines of Agricultural Animals for Research and Teaching at Zhejiang University.

\section{Isolation of Bone Marrow-Derived Macrophages (BMDMs)}

BMDMs were obtained from the femoral cavity of WT or Lcn $2^{-/}$mice ( $n=6$ per group) after removal of attached muscle tissues. The cells were filtered and cultured in 6-well plates with complete DMEM medium (Gibco, USA) containing 30\% conditioned L929 media (Cell Bank of Chinese Academy of Sciences, China), 20\% FBS (Gibco, USA), penicillin (100 I.U./mL) and streptomycin $(100 \mu \mathrm{g} / \mathrm{mL})$ (Sangon Biotech, China). On day 7 , BMDM cultures with nearly $100 \%$ confluence could be used for further studies. Before treated with LPS, BMDMs plated in 6-well plate were cultured in 10\% FBS DMEM medium without antibiotics. After cells were incubated to approximately $90 \%$ confluent, BMDMs were treated with $2 \mu \mathrm{g}$ of Len 2 for $22.5 \mathrm{~h}$ before adding $1 \mu \mathrm{g}$ of LPS for 1.5 $\mathrm{h}$ at $37^{\circ} \mathrm{C}$. Then all cells were washed by PBS and used for RNA or protein extraction. 


\section{Cell Line Cultivation}

RAW264.7 macrophages were purchased from Cell Bank of the Chinese Academy of Sciences (Shanghai, China) and maintained in DMEM supplemented with $10 \%$ FBS, penicillin $(100 \mathrm{I} . \mathrm{U} . / \mathrm{mL})$ and streptomycin $(100 \mu \mathrm{g} / \mathrm{mL})$ at $37^{\circ} \mathrm{C}$ in humidified air containing $5 \% \mathrm{CO}_{2}$. Cells were seeded in 6-well plates and grown overnight until 90\% confluence, Then cells were washed with PBS and incubated in DMEM medium without antibiotics and FBS for 1 2 h. RAW264.7 macrophages were treated with $2 \mu \mathrm{g}$ of Lcn 2 for $22.5 \mathrm{~h}$ before adding $1 \mu \mathrm{g}$ of LPS for $1.5 \mathrm{~h}$ at $37^{\circ} \mathrm{C}$ and used for further determination.

\section{Histomorphology Analysis}

Tissues of liver, jejunum and ileum were fixed with $4 \%$ paraformaldehyde and embedded in paraffin (Google Biotech, China). The sections were $5 \mu \mathrm{m}$ thick and stained with hematoxylin and eosin (H\&E) (NanJing KeyGen Biotech, China). Images were captured by a DM3000 microscope (Leica, Wetzlar, Germany).

\section{Immunofluorescence Staining}

Liver, jejunum and ileum were fixed in $4 \%$ paraformaldehyde overnight followed by paraffin embedding. Then $3-\mu \mathrm{m}$ tissue sections were deparaffinized in xylene (Google Biotech, China) and rehydrated in a series of graded ethanol solutions. After washing with PBS, sections were immersed in $0.01 \mathrm{M}$ citric acid buffer at $98-100^{\circ} \mathrm{C}$. Then cooled sections were blocked with $20 \%$ normal sheep serum, and stained using rabbit polyclonal antibody Len2 (diluted at 1:250, Abcam, USA) and Alexa Fluor 488 goat anti-rabbit polyclonal secondary antibody IgG (diluted at 1:400, Abcam, USA). For immunofluorescence co-localization analysis of Lcn2 and macrophage, sections were stained using rabbit polyclonal antibody Len2 (diluted at 1:250), rat monoclonal antibody F4/80 (diluted at 1:250, Abcam, USA), Alexa Fluor 488 goat anti-rabbit polyclonal secondary antibody $\operatorname{IgG}$ (diluted at 1:400) and Alexa Fluor 647 goat anti-rat polyclonal secondary antibody IgG (diluted at 1:400, Abcam, USA). For immunofluorescence analysis for inducible nitric oxide synthase (iNOS) determination, RAW264.7 macrophages were fixed in $4 \%$ paraformaldehyde, washed with PBS and then permeabilized with ice-cold $0.5 \%$ Triton X-100 (NanJing KeyGen Biotech, China). The cells were blocked with PBS containing 10\% bovine serum albumin (NanJing KeyGen Biotech, China), and then incubated with rabbit monoclonal antibody iNOS (diluted at 1:250, Abcam, USA) and Alexa Fluor 488 goat anti-rabbit IgG (diluted at
1:400). Finally the sections were stained with $50 \mathrm{mg} / \mathrm{mL}$ of 4',6-diamidino-2- phenylindole (DAPI) (Boster Biological Technology, USA) and imaged by confocal laser scanning microscope (LSM-510 Meta, Zeiss, Germany).

\section{ELISA Determination}

The levels of cytokines including interleukin (IL)-1 $\beta$, IL-6 and IL-10 in serum of mice were determined by Mouse ELISA kits (eBioscience, USA) according to the manufacturer's instructions.

\section{Western Blot Analysis}

Liver or intestinal tissues were lysed using M-PER mammalian protein extraction reagent supplemented with HALT proteinase and phosphatase inhibitor cocktails (Thermo-fisher Scientific, USA), Protein concentrations were determined by Bradford protein assay with BSA standards (Bio-Rad). Total protein was extracted and equal quantities of proteins $(10 \mu \mathrm{g})$ were electrophoresed on 10\% SDS-PAGE, and then transferred onto PVDF membranes (Sangon Biotech, China). The membrane was incubated with primary antibodies and then with horseradish peroxidase (HRP)-conjugated secondary antibody. ECL Detection System (CliNX, Shanghai, China) was used to detect antibody reactivity. Primary antibodies included rabbit monoclonal antibody $\beta$-actin (Abcam, USA) and rabbit polyclonal antibody Len2 (Abcam, USA), which were all diluted at 1:1000. Appropriate second antibodies (Cell Signaling Technology, USA) were all diluted at 1:2000.

\section{Gene Expression Analysis}

Total RNAs were extracted from cells and animal tissues by Trizol Reagent according to the manufacturer's instructions (Sigma Aldrich, USA). The concentration of RNA was determined by NanoDrop ND-1000 (Thermo-fisher Scientific, USA). One microgram of total RNA was converted to cDNA using an M-MuLV kit (Thermo Fisher Scientific, USA) in combination with random hexamer primers. Real-time PCR was performed using the FastStart Universal SYBR Green Master fluorescence quantitative kit (Thermo Fisher Scientific, USA) on an Applied Biosystems SteponeTM plus Real-Time PCR System. All the data were normalized to the housekeeper gene GAPDH from the same individual sample. The $2^{-\Delta \Delta \mathrm{Ct}}$ method was used to determine the relative mRNA expression levels. Specific primers sequences are listed in Table 1. 
Table I Primer Sequences for the Real-Time PCR Amplification

\begin{tabular}{|c|c|c|}
\hline Gene & Primer $\left(5^{\prime} \rightarrow 3^{\prime}\right)$ & Accession Number \\
\hline GAPDH & $\begin{array}{l}\text { F: TGCGACTTCAACAGCAACTC } \\
\text { R: GCCTCTCTTGCTCAGTGTCC }\end{array}$ & NM_008084.3 \\
\hline IL-I $\beta$ & $\begin{array}{l}\text { F: AGTTGACGGACCCCAAAAG } \\
\text { R: TTTGAAGCTGGATGCTCTCAT }\end{array}$ & NM_008361.4 \\
\hline TNF- $\alpha$ & $\begin{array}{l}\text { F: GCTCTTCTGTCTACTGAACTTCGG } \\
\text { R: ATGATCTGAGTGTGAGGGTCTGG }\end{array}$ & NM_013693.3 \\
\hline IL-6 & $\begin{array}{l}\text { F: CCCCAATTTCCAATGCTCTCC } \\
\text { R: CGCACTAGGTTTGCCGAGT }\end{array}$ & NM_03 I 68.2 \\
\hline iNOS & $\begin{array}{l}\text { F: CTCACCTACTTCCTGGACATTAC } \\
\text { R: CAATCTCTGCCTATCCGTCTC }\end{array}$ & NM_010927.4 \\
\hline TGF- $\beta$ I & $\begin{array}{l}\text { F: GTCACTGGAGTTGTACGGCA } \\
\text { R: AGCCCTGTATTCCGTCTCCT }\end{array}$ & NM_011577.2 \\
\hline Arg-1 & $\begin{array}{l}\text { F: CCTGGAACTGAAAGGAAAG } \\
\text { R: TTGGCAGATATGCAGGGAGT }\end{array}$ & NM_007482.3 \\
\hline IL-10 & $\begin{array}{l}\text { F: TGGGTTGCCAAGCCTTATCG } \\
\text { R: TTCAGCTTCTCACCCAGGGA }\end{array}$ & NM_010548.2 \\
\hline MIP-2 & $\begin{array}{l}\text { F: CACTCTCAAGGGCGGTCAAA } \\
\text { R: GGTTCTTCCGTTGAGGGACA }\end{array}$ & NM_009|40.2 \\
\hline MCP-I & $\begin{array}{l}\text { F: GATGCAGTTAACGCCCCACT } \\
\text { R: ACCCATTCCTTCTTGGGGTC }\end{array}$ & NM_011333.3 \\
\hline Lcn2 & $\begin{array}{l}\text { F: ACATTTGTTCCAAGCTCCAGGGC } \\
\text { R: CATGGCGAACTGGTTGTAGTCCG }\end{array}$ & NM_00849I.I \\
\hline
\end{tabular}

\section{Transmission Electron Microscopy (TEM) Analysis}

Tissues were fixed in $2.5 \%$ glutaraldehyde overnight, washed with PBS and fixed with 1\% OsO4 (NanJing KeyGen Biotech, China) for $1 \mathrm{~h}$. The samples were then dehydrated in a series of graded ethanols and then transferred into acetone. Then samples were placed in a mixture of acetone and Spurr resin (Head Biotechnology, China), and transferred to Spurr resin mixture overnight. The tissue sections were placed in capsules containing the embedding medium (NanJing KeyGen Biotech, China) and heated for $9 \mathrm{~h}$ at $70^{\circ} \mathrm{C}$. The sections were then stained with uranyl acetate and alkaline lead citrate (NanJing KeyGen Biotech, China) for $15 \mathrm{~min}$ and observed by TEM (Model H-7650, HITACHI, Japan).

\section{Flow Cytometry Analysis for Phagocytosis of Macrophages}

BMDMs were seeded in a 6-well plate until $90 \%$ confluent, and treated with $0.5 \mathrm{mg} / \mathrm{mL}$ of FITC-dextran (Sigma Aldrich, USA) for $2 \mathrm{~h}$ at $37^{\circ} \mathrm{C}$. Then cells were digested by trypsin, collected by centrifuge at $1000 \mathrm{~g}$ for $5 \mathrm{~min}$, and finally resuspended with $500 \mu \mathrm{L}$ PBS, which could be used for flow cytometry (MACSQuant VYB, Germany) analysis. The phagocytosis of macrophages was expressed as mean fluorescence intensity.

\section{Proteomics}

BMDMs from WT and Lcn $2^{-/}$treated with LPS $(1 \mu \mathrm{g} / \mathrm{mL})$ for $1.5 \mathrm{~h}$ were lysed in SDT lysis buffer (4\% (w/v) SDS, $100 \mathrm{mM}$ Tris/HCL, 0.1M DTT, pH7.6). Samples were centrifuged to remove debris, and then the supernatant was collected. For each sample, protein was digested by trypsin (mass ratio 1:50 trypsin-to-protein). Peptide fragments were desalted by $\mathrm{C} 18$ Cartridge, freeze-dried, redissolved by $40 \mu \mathrm{L}$ of $0.1 \%$ formic acid solution, and then quantified (OD280). The samples were then analyzed using nano-flow liquid chromatography electrospray tandem mass spectrometry (nanoLC-ESI-MS/MS) with a Thermo Scientific Q-Exactive Mass Spectrometer (Germany) coupled to a Thermo Ultimate 3000 RSLCnano HPLC system. Peptide fragments were selected for MS/MS by normalized collision energy (NCE) and detected in the orbitrap at a resolution of 17,500. Raw database was performed using MaxQuant search engine (v. 1.5.3.17). Gene Ontology (GO) annotation and Kyoto Encyclopedia of Genes and Genomics (KEGG) pathway research were conducted to analyze quantitative protein. Proteins with P-value $<0.05$ and an absolute $\log _{2}$ fold change $(\mathrm{FC}) \geq 1.6$ or $\leq 0.625$ were considered significantly. The whole experiment strategy was developed for quantitative proteomic profiles of BMDMs with 3 biological replicates.

\section{Statistical Analysis}

Statistical analyses of experimental data were performed using GraphPad Prism software (version 8, GraphPad Software Inc., San Diego, CA, USA). The results are expressed as mean \pm standard error of the mean (SEM). The difference between groups was determined by Student's $t$ test or, if there were more than two groups, by one-way analysis of variance followed by Duncan's test. The test was independently repeated at least three times. $\mathrm{P}$ values of $<0.05$ was recognized as statistically significant. ${ }^{*} \mathrm{P}<0.05,{ }^{*} \mathrm{P}<0.01$.

\section{Results LPS Challenge Induces Lcn2 Expression}

To identify that Lcn2 is one of the acute-phase proteins during inflammation, we intraperitoneally injected LPS into WT mice and analyzed the expression of Lcn2 in tissues. Fluorescence staining demonstrated that the tremendous levels of Lcn2 
protein were induced in liver, jejunum and ileum at $24 \mathrm{hpi}$ (Figure 1A). Western blot analysis showed that Lcn2 expression was at least 1.3 -fold increased at $8 \mathrm{hpi}$ in three detected tissues. Lcn 2 levels in the liver were increased 28 -fold at $24 \mathrm{hpi}$ (Figure 1B). Notably, Lcn2 protein levels were significantly (P $<0.01$ ) elevated and peaked by $24 \mathrm{hpi}$ or $32 \mathrm{hpi}$, and then declined (Figure 1B-D).

\section{Lcn2 Deficiency Results in Increased Inflammation}

To determine the role of elevated Lcn2 during LPS-induced inflammation, we assessed the effect of Lcn2 on systemic inflammation using $\mathrm{Lcn} 2^{-/-}$mice. LPS challenge initially caused diarrhea, resulting in rapid body weight loss and low food intake. Food intake increased after 24 hpi both in Lcn $2^{-/-}$ mice and WT mice, but the body weight is only increasing in WT mice (data now shown). When compared to WT mice, TEM analysis showed that the microvilli of $\mathrm{Lcn} 2^{-/-}$mice was fragmentary, and the number and height of microvilli were decreased apparently (Figure 2A). After LPS challenge, inflammatory cytokines were all increased at once, peaked by 8 hpi and then declined (Figure 2B and C). When compared with WT mice after LPS challenge, serum levels of proinflammatory cytokines such as IL-1 $\beta$ and IL-6 were significantly $(\mathrm{P}<0.05)$ higher at $8 \mathrm{hpi}$ to $32 \mathrm{hpi}$ in $\mathrm{Lcn} 2^{-/-}$mice (Figure 2B and $\mathrm{C}$ ), while that of anti-inflammatory cytokine IL-10 was significantly $(\mathrm{P}<0.05)$ lower at 8 hpi (Figure $2 \mathrm{D})$. The mRNA expression levels of IL-1 $\beta$ in indicated tissues (liver, jejunum and ileum) also exhibited the similar expression pattern at different time points after LPS challenge (Figure 2E).

\section{Lcn2 is Predominantly Located in Macrophages During Inflammation}

In order to determine the major cell sources that promote the production of Lcn2 during LPS-induced inflammation, we next performed double immunofluorescence staining analysis on liver sections of WT mice at $24 \mathrm{~h}$ after LPS challenge. As expected, the confocal images showed that LPS induced high expression of Lcn 2 in hepatocytes (Figure 3A). Interestingly, Lcn2 was highly expressed in Kupffer cells, which are specialized macrophages located in the liver. Subcellular localization analysis further confirmed that Lcn2 was mainly expressed in the cytoplasm of RAW264.7 macrophages (Figure 3B). These findings suggested that macrophages might be the main source for elevated Lcn2 production during LPS-induced inflammation.

\section{Lcn2 Deficiency Enhances the Pro-Inflammatory Response of Macrophages}

Macrophages are the main producers of inflammatory cytokines. The above results illustrated that Lcn2 was predominantly expressed in macrophages, and its deficiency could aggravate the inflammation. The observed elevation of proinflammatory cytokines in $\mathrm{Lcn} 2^{-/-}$mice may also be due to the fact that macrophages require Lcn 2 to effectively modulate the balance between pro-inflammatory and anti-inflammatory responses during systemic inflammation. In order to test this hypothesis directly, we isolated BMDMs from mice and examined the expression levels of various inflammatory cytokines after LPS treatment in vitro. BMDMs exhibited an irregular shape after a week of cell culture, which marked its differentiation into mature macrophages. However, there are no significantly morphological differences between BMDMs from WT and $\mathrm{Lcn} 2^{-/-}$mice and there was no Lcn2 expression in BMDMs of $\operatorname{Lcn} 2^{-/}$mice, and LPS induced Lcn 2 expression in BMDMs from WT mice (data not shown). After treated with LPS, BMDMs from $\mathrm{Lcn} 2^{-/-}$mice showed significantly $(\mathrm{P}<$ $0.01)$ higher mRNA levels of pro-inflammatory cytokines including IL-1 $\beta$ (Figure 4A), TNF- $\alpha$ (Figure 4B) and iNOS (Figure 4D) when compared with WT mice. Lcn2 deficiency also increased the mRNA expression of IL-6, but the difference was not significant even in LPS stimulation $(\mathrm{P}=0.084)$ (Figure 4C). Conversely, the transcripts of anti-inflammatory cytokines, such as transforming growing factor $\beta 1$ (TGF- $\beta 1$ ), IL-10 and arginase-1 (Arg-1) were strongly $(\mathrm{P}<0.01)$ repressed in BMDMs from $\mathrm{Lcn}^{-/}$mice (Figure $4 \mathrm{E}-\mathrm{G}$ ). Moreover, the mRNA levels of chemokines macrophage inflammatory protein-2 (MIP-2) and monocyte chemoattractant protein-1 (MCP-1) related macrophages migration in BMDMs from $\mathrm{Lcn} 2^{-/-}$mice were both significantly $(\mathrm{P}<$ 0.05 ) reduced (Figure $4 \mathrm{H}$ and I). Thus, the results indicated that Lcn2 played an important role in the expression regulation of inflammatory cytokines by macrophages during LPSinduced inflammation.

\section{Lcn2 Attenuates the Inflammatory Response of Macrophages}

Since BMDMs from $\mathrm{Lcn}^{-/}$mice exhibited enhanced proinflammatory response, we then want to test whether the addition of Lcn 2 could help alleviate the inflammatory response of BMDMs. Upon stimulation with LPS, the transcript levels of pro-inflammatory cytokines were all significantly $(\mathrm{P}<0.01)$ attenuated (Figure 4A-D) after pre-treatment with the 
A
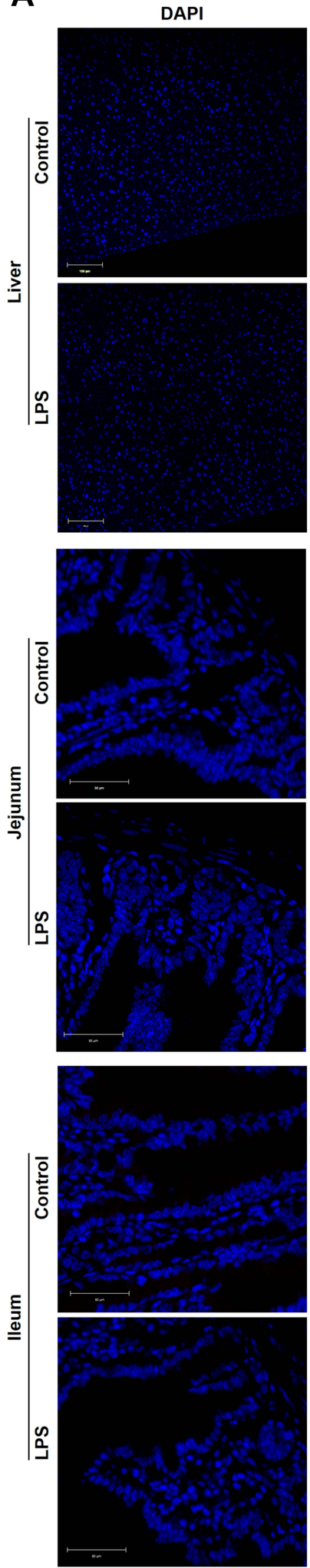

Lcn2
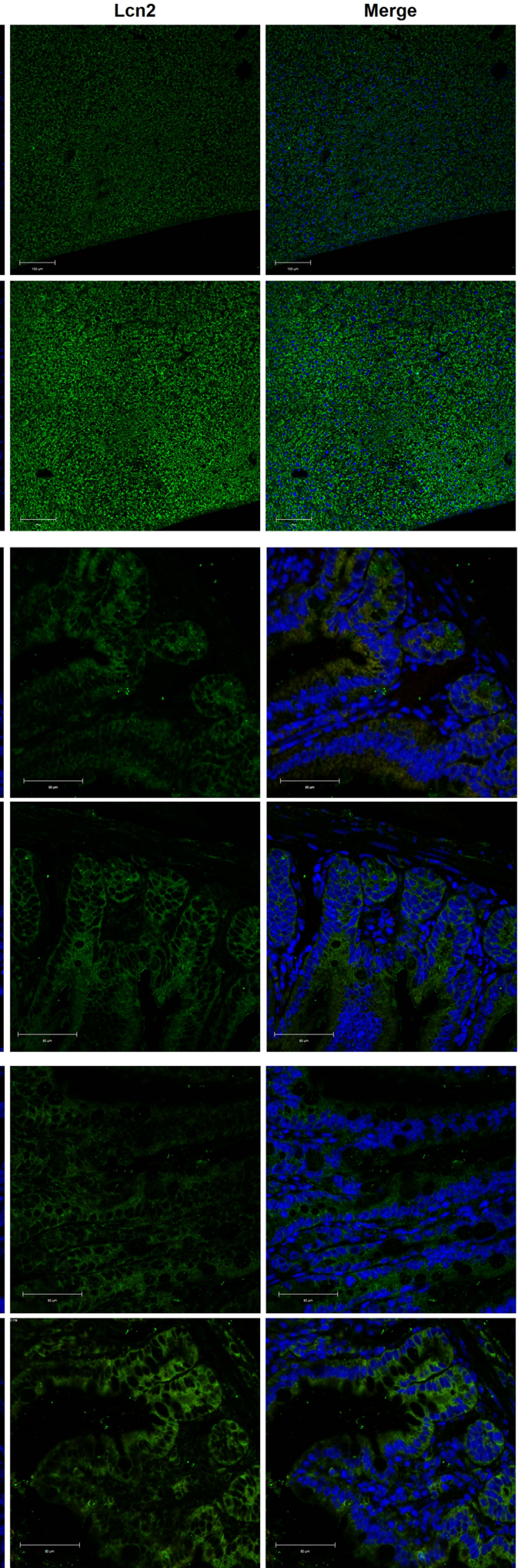

Merge

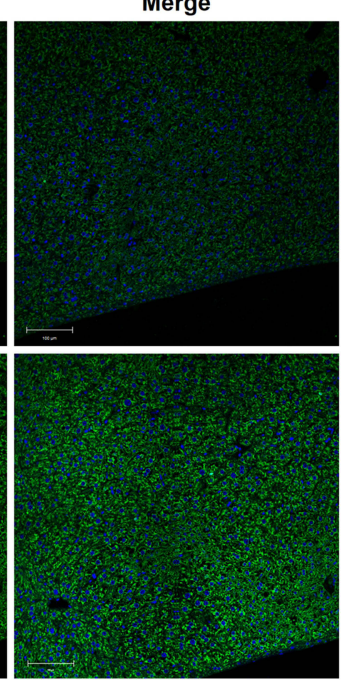

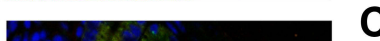

C
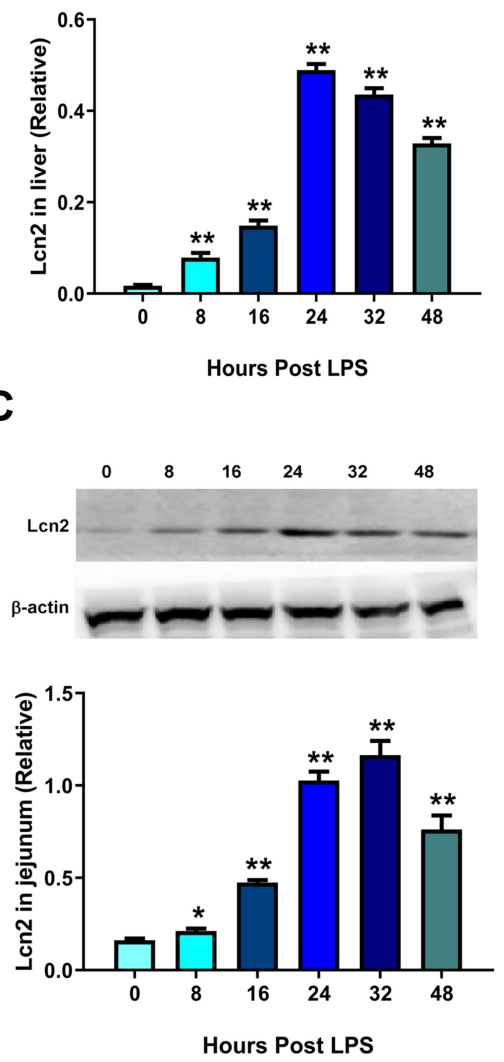

D
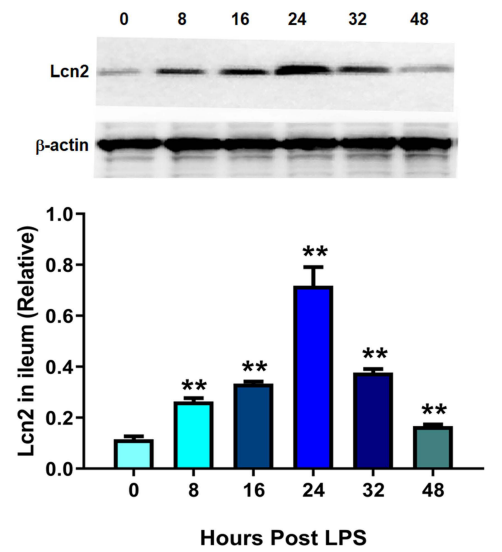

Figure I LPS challenge induces Lcn2 expression. WT mice were intraperitoneally injected with saline (Control) or $2 \mu \mathrm{g} / \mathrm{g}$ LPS. (A) Representative confocal images of Lcn2 expression in the liver, jejunum and ileum of mice at $24 \mathrm{~h}$ post LPS injection $(n=4)$. DAPI, blue; Lcn2, green. (B-D) Protein levels of Lcn2 in indicated tissues from WT mice after LPS challenge at different time points were examined by Western blot $(n=4)$. Values were average means of triplicate experiments. Results were expressed as means \pm SEM. Statistical analysis used Mann-Whitney U-tests. $* \mathrm{P}<0.05$, $* * \mathrm{P}<0.01$. 
A
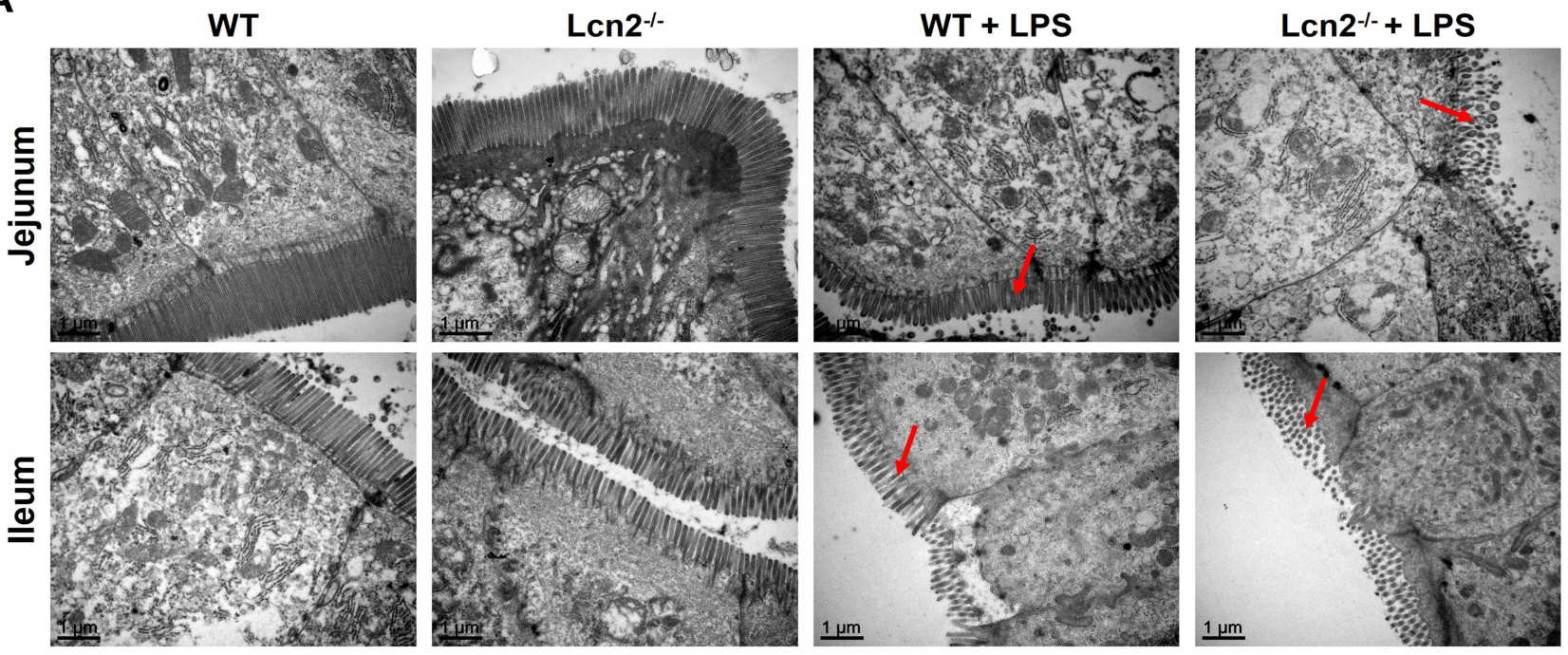

B

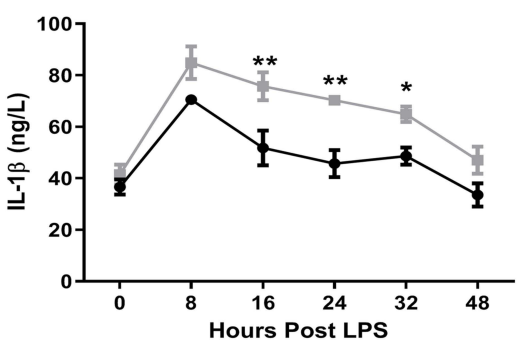

E

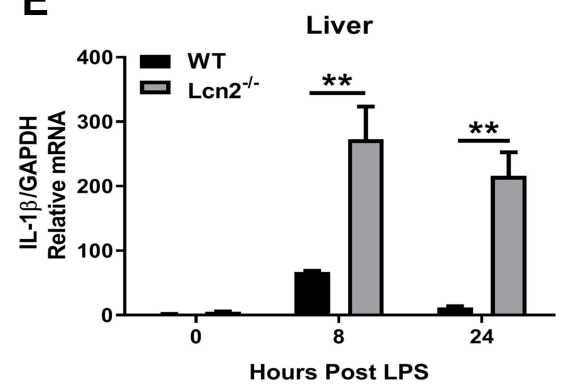

C
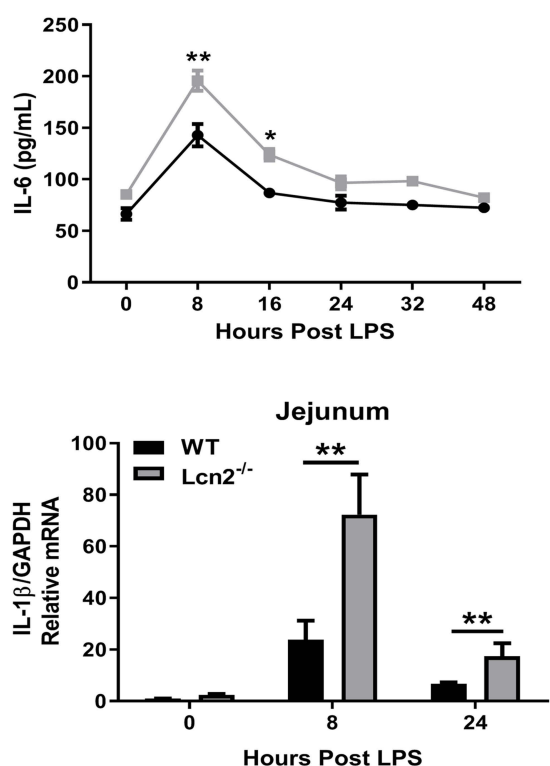

D
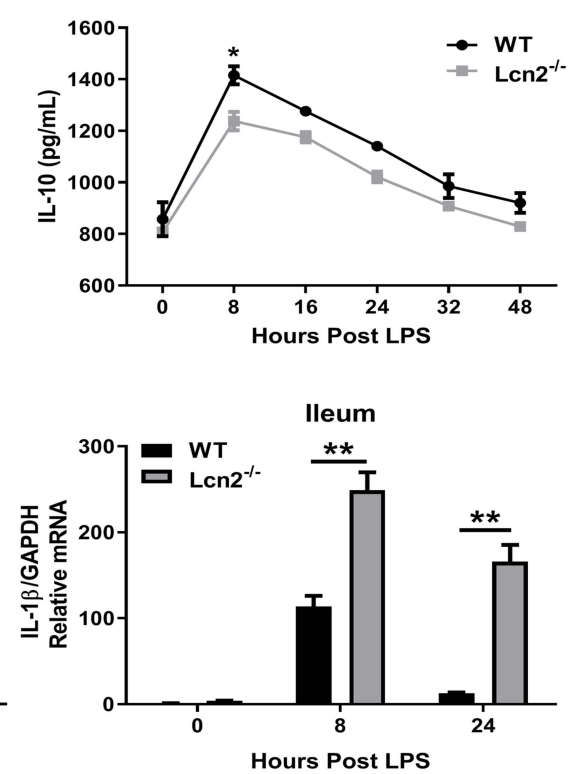

Figure 2 Lcn2 deficiency results in increased inflammation. WT and Lcn $2^{-/-}$mice were intraperitoneally injected $2 \mu g / g$ LPS. (A) Representative TEM images of microvilli morphology in jejunum and ileum of mice at $24 \mathrm{hpi}(n=4)$. (B-D) Protein levels of IL-I $\beta$, IL-6 and IL- I0 were examined by ELISA from serum of mice at 0,8 , I6, 24,32 and 48 hpi. (E) Relative mRNA expression of IL-I $\beta$ normalized to GAPDH was examined by qRT-PCR from liver, jejunum and ileum at 0,8 and 24 hpi $(n=4)$. The mRNA expression of IL-I $\beta$ in WT mice at 0 hpi was used as the control in each tissue. Values were average means of triplicate experiments. Results were expressed as means \pm SEM. Statistical analysis used Wilcoxon signed-rank test. $* \mathrm{P}<0.05$, $* * \mathrm{P}<0.01$.

recombinant Lcn2, but those of anti-inflammatory cytokines were all significantly $(\mathrm{P}<0.05)$ promoted (Figure $4 \mathrm{E}-\mathrm{G})$. We then employed mice RAW264.7 macrophages for further verification. Similarly, after LPS treatment, the mRNA expression of pro-inflammatory factors such as TNF- $\alpha(p<0.05)$, IL-6 (P $<0.01)$, and IL-1 $\beta(\mathrm{P}<0.01)$ were all strongly repressed with the pre-treatment of recombinant Lcn2 (Figure 5A-C), while transcription levels of anti-inflammatory cytokine IL-10 and chemokine MCP-1 were both markedly $(\mathrm{P}<0.05)$ upregulated (Figure 5D and E). Consistent with the results of transcription, immunofluorescence analysis showed that the expression of iNOS was significantly $(\mathrm{P}<0.01)$ depressed by adding Lcn2 (Figure 5F and G). Therefore, Lcn2 could alleviate the inflammation response of both BMDMs and RAW264.7 macrophages.

\section{Proteomic Analysis of LPS-Treated BMDMs from Lcn2 ${ }^{-/-}$Mice}

In order to comprehensively investigate the molecular mechanism by which $\operatorname{Lcn} 2$ regulates the function of 
A
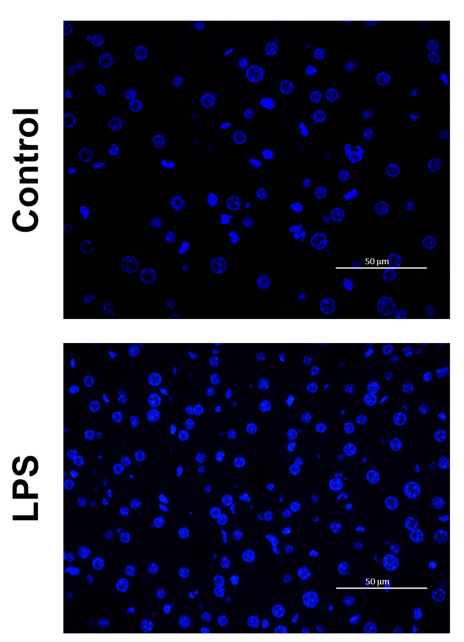

Len2
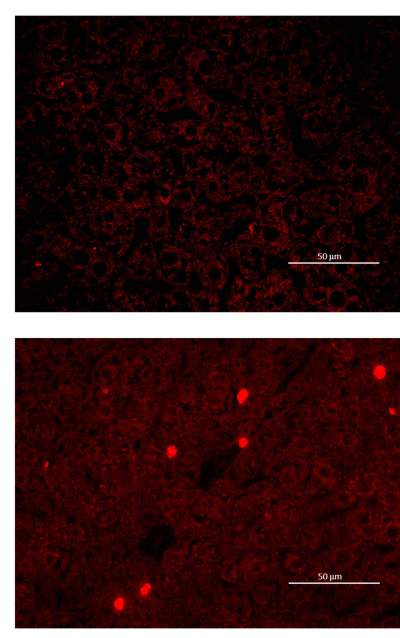

$F 4 / 80$
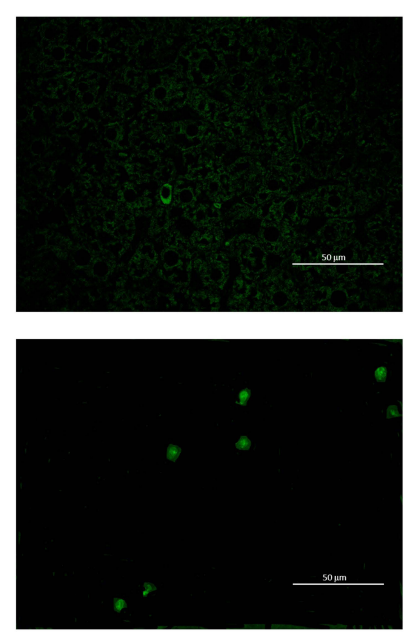

Merge
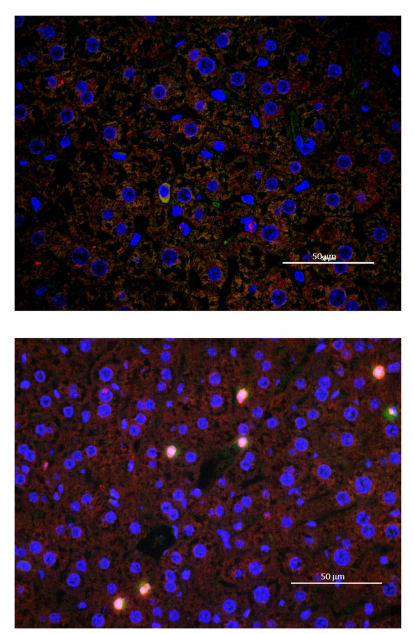

B

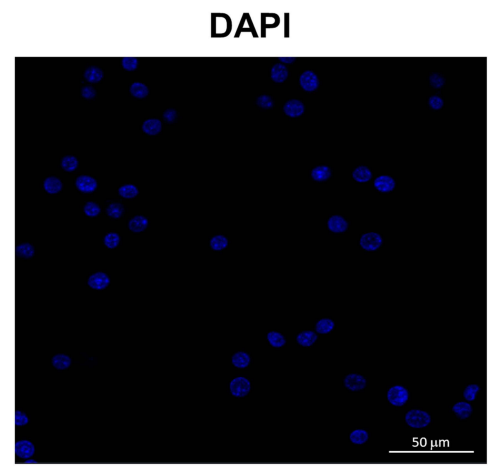

Len2

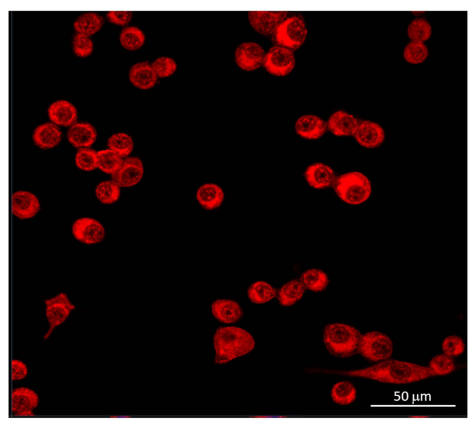

Merge

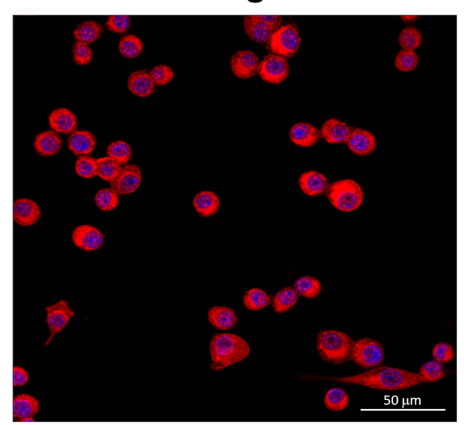

Figure 3 Lcn2 is predominantly expressed in macrophages during LPS-induced inflammation. (A) Representative confocal images of Lcn2 and macrophages in the liver at 24 h post LPS injection $(n=4)$. DAPI, blue; Lcn2, red; macrophage marker F4/80, green; Merge, yellow. (B) Representative confocal images of Lcn2 in RAW264.7 macrophages $(n=6)$. DAPI, blue; Lcn2, red.

macrophages, we performed mass spectrometry (MS)-based quantitative proteomic analysis of BMDMs from WT and $\mathrm{Lcn}^{-/-}$mice with LPS. In total, 4068 proteins were identified, among which 2993 proteins were quantified with three biological replicates. Differentially expressed proteins were identified by a cutoff of absolute folding change $\geq 1.6$ or $\leq$ 0.625 , and $p$ value $<0.05$. The results showed that 144 proteins were differentially expressed between BMDMs from WT and $\mathrm{Lcn} 2^{-/}$mice after LPS challenge. Among them, 47 proteins in BMDMs of $\mathrm{Lcn}^{-/-}$mice were significantly higher and 97 proteins were much lower than in BMDMs of WT mice (Figure 6A). The data also showed that in LPS-treated BMDMs of Lcn $2^{-/-}$mice, the expression of pro-inflammatory factor-related proteins, such as IL-1 $\beta$, apoptosis-associated speck-like protein containing a CARD (PYCARD) and the nuclear factor kappa-B (NF-kB), was higher, while that of anti-inflammatory factor-related proteins, such as immunity-related GTPase family $\mathrm{M}$ protein 1 (Irgm1) and $\mathrm{I} \kappa \mathrm{B} \alpha$ was lower (Figure 6B), which was consistent with above results in mice. PYCARD, a key mediator in apoptosis and inflammation, was up-regulated 8.0-fold in BMDMs of $\mathrm{Lcn} 2^{-/-}$mice as compared to those of WT mice. However, Irgm1, a positive regulator of both pro-inflammatory cytokine production and macrophage motility, was down-regulated 0.42 -fold in BMDMs of $\mathrm{Lcn}^{-/-}$mice as compared to those of WT mice (Figure 6B). GO analysis revealed that BMDMs of WT mice were highly enriched in the proteins involved in several biological processes related to the regulation of immune response, such as immune system process, cellular process, metabolic process, biological regulation, as well as response to external stimulus (Figure 6C). And binding and catalytic activity were the most highly enriched pathways involving Len2 (Figure 6D). 

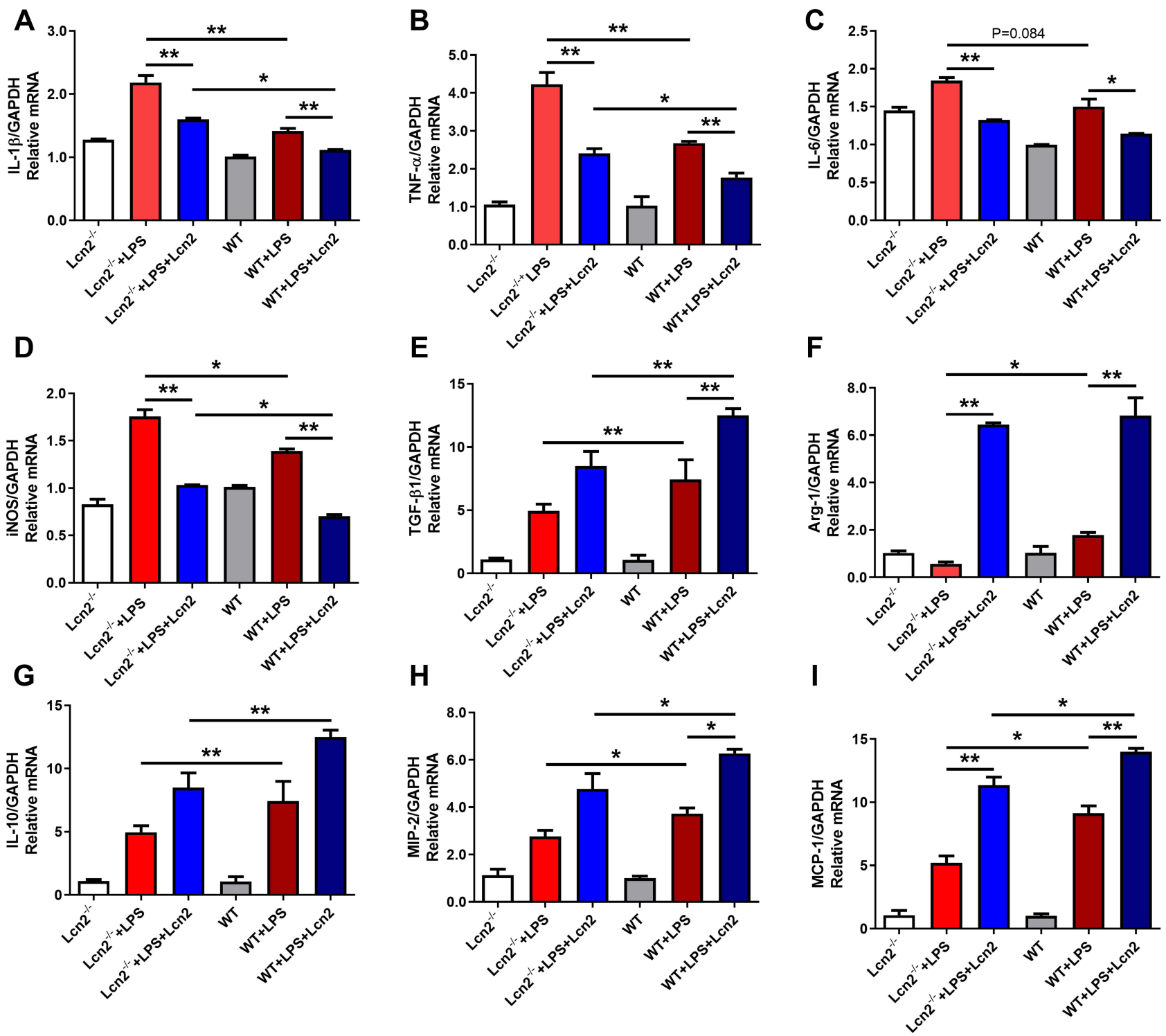

Figure 4 Lcn2 deficiency enhances the pro-inflammatory response of macrophages. (A-I) Quantitative determination of cytokines mRNA expression levels in the LPStreated BMDMs from WT and Lcn2 ${ }^{-1-}$ mice. Relative mRNA expression of cytokines normalized to GAPDH rRNA as the reference genes. The mRNA expression of cytokines in WT mice was used as the control. Values were average means of triplicate experiments with two mice used for the isolation of BMDMs per genotype per experiment $(n=2)$. Results were expressed as means \pm SEM. Statistical analysis used Wilcoxon signed-rank test. $* \mathrm{P}<0.05, * * \mathrm{P}<0.01$.

Lcn2 Deficiency Inhibits the Phagocytosis of BMDMs and Hepatocyte Autophagy

Since Lcn2 deficiency significantly suppressed the expression of proteins associated with binding activity (Figure 6B), we then further investigated whether Lcn2 would affect the migration and endocytosis of macrophages. Bioinformatics enrichment analysis from proteomic analysis showed that revealed proteins that inhibit macrophage motility and phagocytosis, such as stathmin and calponin-2, were up-regulated in LPS-treated BMDMs of $\mathrm{Lcn} 2^{-/-}$mice as compared to those of WT mice (Figure 7A). On the contrary, proteins that facilitate phagocytosis including reticulocalbin-1, lactadherin (Mfge8), and tapasin were all down-regulated significantly in LPS-treated BMDMs of $\mathrm{Lcn}^{-/-}$mice. Furthermore, flow cytometry analysis confirmed that Lcn2 deficiency significantly $(\mathrm{P}<0.01)$ reduced the phagocytosis rate of FITC-Dextran by $75 \%$ in BMDMs (Figure 7B). In addition, GO analysis also revealed that some differentially expressed proteins are related to autophagy, such as PYCARD and Irgm1 (Figure 7C). TEM analysis showed that there were more autophagosomes (a nearly round vacuole in the cytoplasm containing lysosomal dissolved impurities) in livers of WT mice, while fewer autophagy 


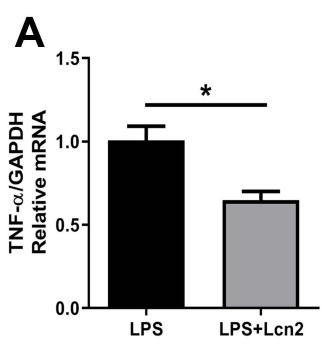

$\mathbf{F}$
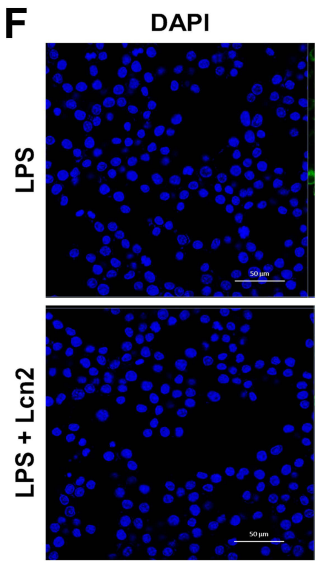

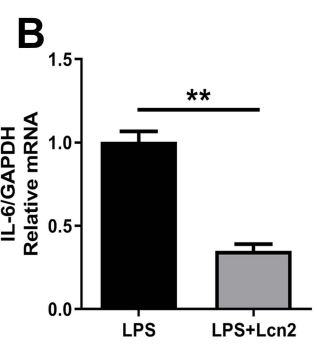

iNOS
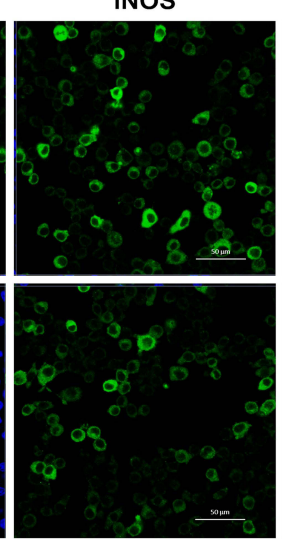
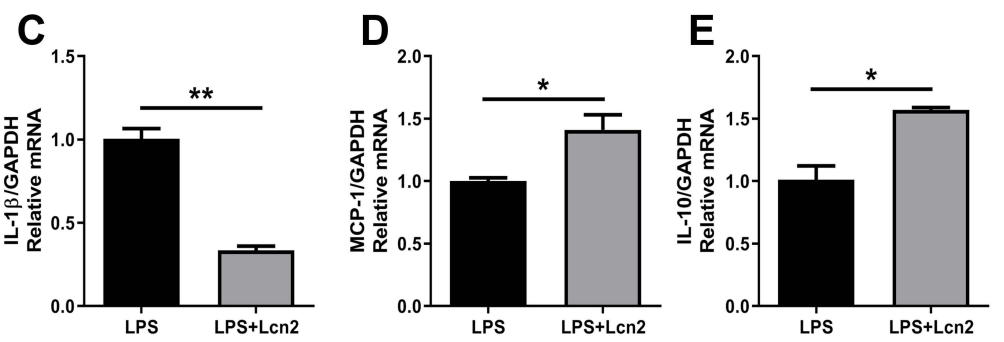

G
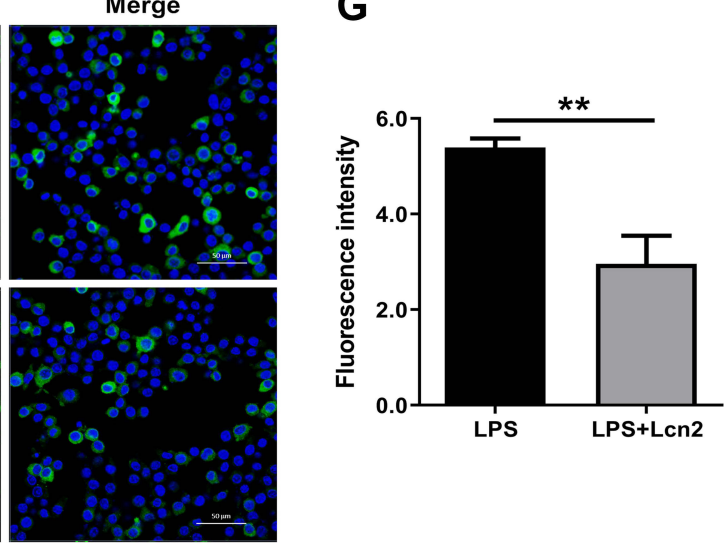

Figure $5 \mathrm{Lcn} 2$ attenuates the inflammatory response of macrophages. RAW264.7 macrophages were treated with $2 \mu \mathrm{g} / \mathrm{mL}$ recombinant Lcn2 for $22.5 \mathrm{~h}$ and I $\mu \mathrm{g} / \mathrm{mL} \mathrm{LPS}$ for I.5 h. (A-E) Real-time PCR analysis of cytokine mRNA expression levels $(n=3)$. Relative mRNA expression of cytokines normalized to GAPDH rRNA as the reference genes. The mRNA expression of cytokines in LPS-treated mice was used as the control. (F-G) RAW264.7 macrophages were subjected to staining with rabbit monoclonal antibody iNOS and Alexa Fluor 488 goat anti-rabbit lgG, and observed by laser scanning confocal microscope $(n=3)$. Cells were assayed for expression by detection of iNOS gene (green) by fluorescence microscopy. Cell nuclei were labeled with DAPI (blue). The number of cells were quantified to the same level for detection of fluorescence intensity. Values were average means of triplicate experiments. Results were expressed as means \pm SEM. Statistical analysis used Wilcoxon signed-rank test. $* \mathrm{P}$ $<0.05, * * \mathrm{P}<0.01$.

area was detected in $\mathrm{Lcn} 2^{-/-}$mice (Figure 7D). Above results indicated that Lcn2 was involved in the phagocytosis function of macrophages, and may play a role in cell autophagy.

\section{Discussion}

Lcn2 is widely known to play a variety of biological roles in iron transport, bacteriostasis, apoptosis and cell survival. However, the specific role of Lcn2 in different inflammatory conditions is not always consistent. For example, Lcn2 was reported to play a pro-inflammatory role in autoimmune encephalomyelitis models of neuroinflammation ${ }^{17}$ and central nervous system. ${ }^{18}$ In contrast, the anti-inflammatory role of Lcn2 was also reported that the level of IL-6 protein in brain tissues of $\mathrm{Lcn} 2^{-/-}$mice treated with LPS was significantly increased. ${ }^{16}$ Lcn2 reduced the expression of cytokines in LPS-treated RAW264.7 macrophages. ${ }^{19}$ Until now, the specific function of Lcn2 in inflammation has not been clarified. In the present study, we provided the first evidence that Lcn2 suppresses inflammatory reactions by mediating the functions of macrophages.
Lcn2 is an acute-phase protein known to be highly upregulated in inflammatory states. ${ }^{13}$ It is thought to be a marker of many inflammatory diseases, and is involved in various types of inflammation. ${ }^{20}$ Previous studies have shown that Lcn2 was significantly elevated in the brain and astrocytes 4 to $8 \mathrm{~h}$ after an inflammatory stimulus. ${ }^{16}$ Similarly, in the present study, we found that Lcn2 protein levels in liver, jejunum and ileum were initially significantly induced at $8 \mathrm{hpi}$, increased to peak by 24 or $32 \mathrm{hpi}$, and then declined, which was induced in a parabolic pattern as well as our previous study of Escherichia coli infection. ${ }^{21}$

In the absence of Lcn2, the mice suffered more severe inflammatory injury including slower recovery of body weight, more swollen liver and spleen, and severe broken intestinal barrier following LPS injection. Pro-inflammatory cytokines IL-1 $\beta$ and IL-6 were both significantly increased in the serum of $\mathrm{Lcn} 2^{-/-}$mice. IL-1 $\beta$ transcripts were also markedly elevated in detected tissues during LPS-induced inflammation. However, anti-inflammatory cytokine IL-10 protein was decreased significantly at $8 \mathrm{hpi}$ in the serum of $\mathrm{Lcn} 2^{-/-}$mice. In accordance with our findings of anti-inflammatory role for Lcn2, significantly elevated levels of multiple pro- 
A

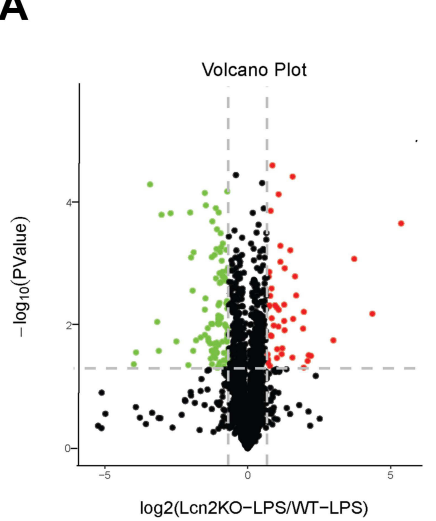

B

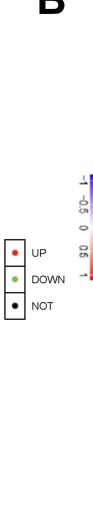

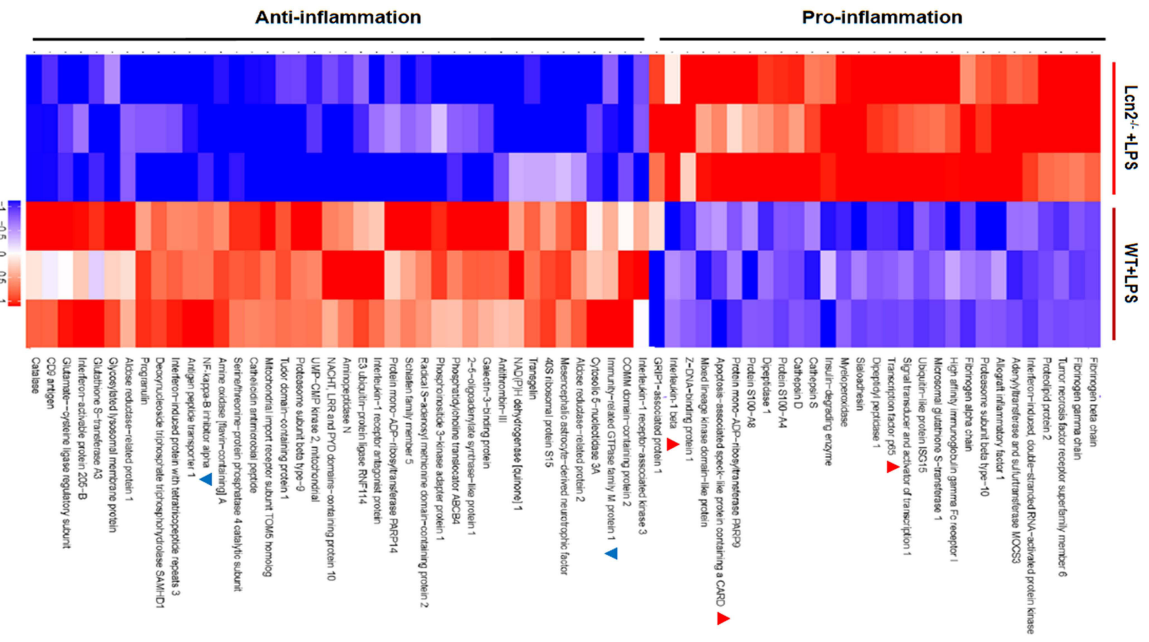

C

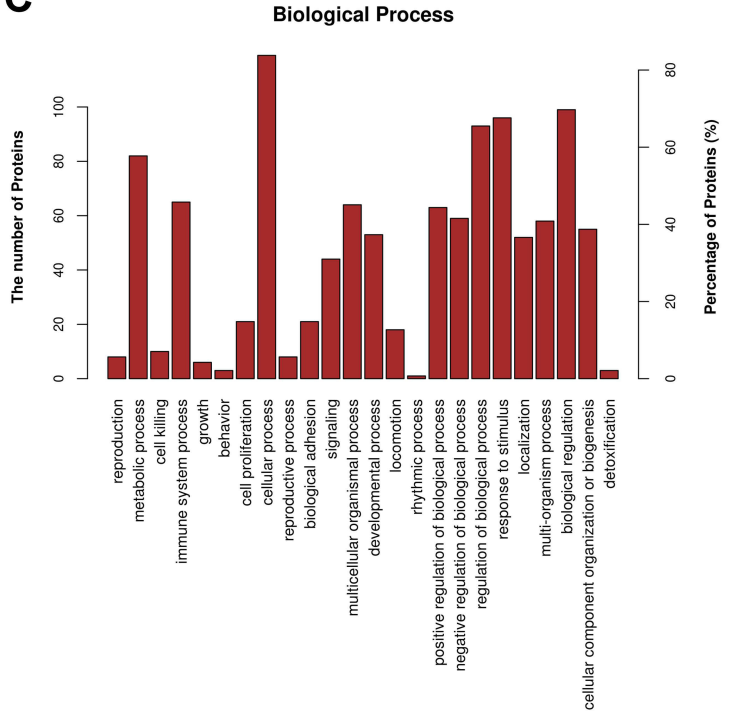

D

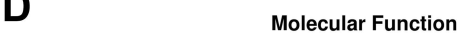

Figure 6 Proteomics analysis of LPS-treated BMDMs from Lcn2 $2^{-/-}$and WT mice. (A) Volcano plots shows that 144 proteins changed significantly (a cutoff of absolutes fold change $\geq 1.6$ or $\leq 0.625$, and $P<0.05$ ). Green dots represent down-regulated proteins, and red dots represent up-regulated proteins. (B) Series of inflammation-related proteins $(n=3)$. Blue color represents down-regulated proteins, and red color represents up-regulated proteins. (C) Biological processes of GO annotation. (D) Molecular function of GO annotation. Bar chart represents biological processes and molecular function enriched by altered proteins in response to Len2 deficiency.

inflammatory molecules were shown in the brain of $\mathrm{Lcn} 2^{-1-}$ mice injected with LPS. ${ }^{16} \mathrm{Lcn} 2$ treatment has also significantly reduced the expression and secretion of TNF- $\alpha$ and IL- 6 in LPS-stimulated Müller cells. ${ }^{22}$ Taken together, these data demonstrated that Lcn2 acted as an important regulator of inflammatory responses in LPS-induced systemic inflammation.

Although bone marrow is normally the primary site of Lcn2 expression, it has been reported that Len2 is highly induced in the liver of bacterial infected mice. ${ }^{18}$ In this study, we demonstrated that $\mathrm{Lcn} 2$ could also be induced in the liver by LPS. Interestingly, we also found that Lcn2 was mainly located in the cytoplasm of macrophages by subcellular localization analysis. Macrophages regulate innate immune response to pathogenic microorganisms and contribute to adaptive immune response, inflammation, and wound repair. During inflammation, circulating monocytes leave the bloodstream and migrate to damaged tissues, where they differentiate into macrophages or dendritic cell populations. ${ }^{23}$ The recruitment of monocytes is essential for the effective control and elimination of bacterial, viral and fungal infections, and contributes to the pathogenesis of inflammation. ${ }^{24}$ Therefore, we next differentiated BMDMs and explored whether alteration of Lcn2 expression would modulate the inflammatory response of macrophages. Similar to our findings of an antiinflammatory role for Lcn2 in vivo, BMDMs from $\mathrm{Lcn} 2^{-/}$ mice showed increased mRNA expression levels of proinflammatory cytokines IL- $1 \beta$, TNF- $\alpha$ and IL-6, while 
A

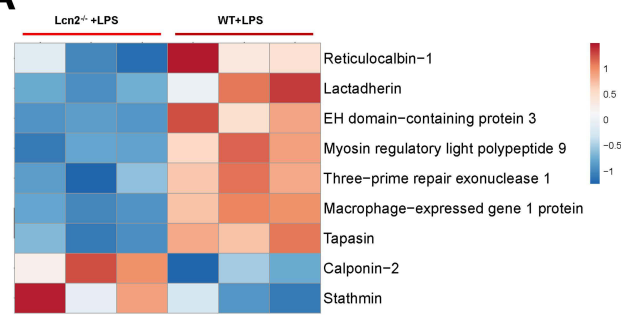

C

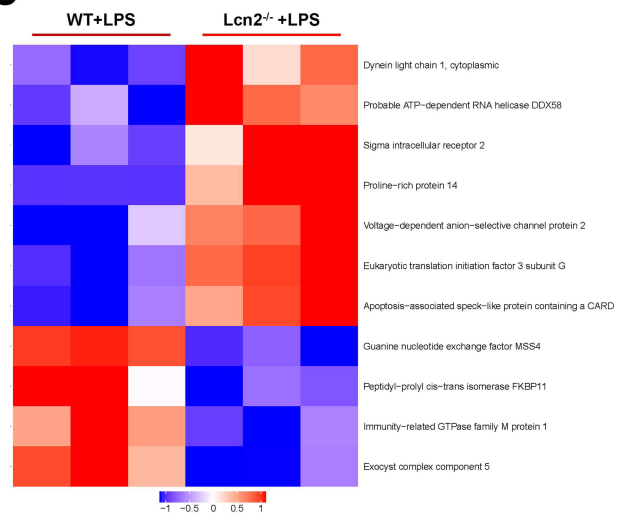

B
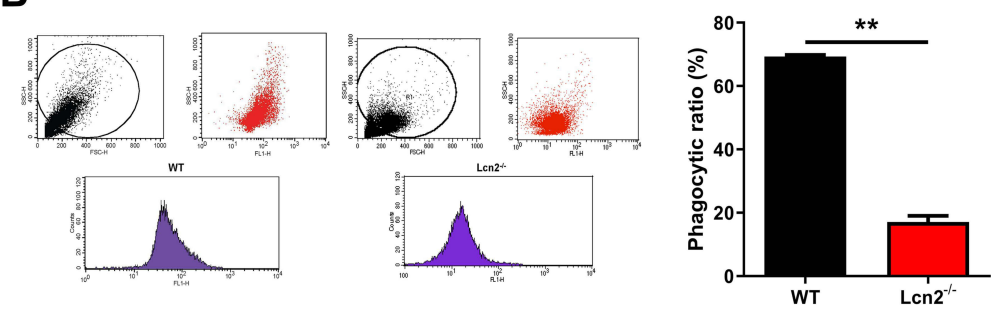

D
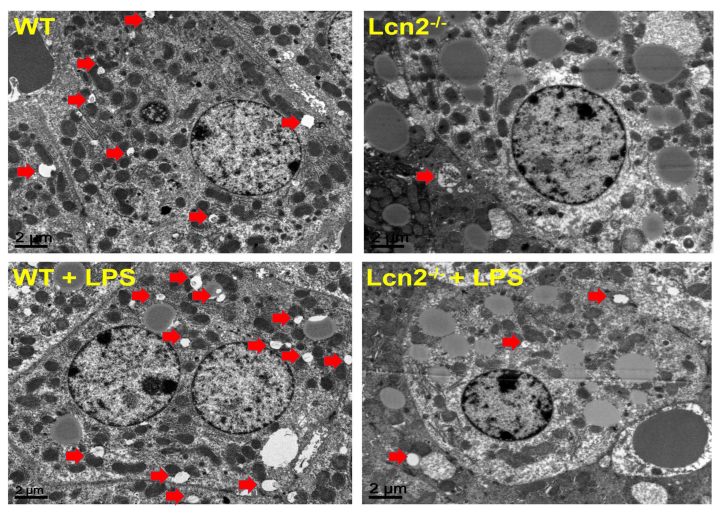

Figure 7 Lcn2 deficiency inhibits the phagocytosis of BMDMs and hepatocyte autophagy. (A) Series of phagocytosis-related proteins $(n=3)$. Blue color represents downregulated proteins, and red color represents up-regulated proteins. (B) Flow cytometry analysis of BMDMs incubated with FITC-dextran ( $n=3$ ). (C) Series of autophagyrelated proteins $(n=3)$. Blue color represents down-regulated proteins, and red color represents up-regulated proteins. (D) Representative TEM images of autophagosomes (red arrows) in liver of mice at 24 hpi $(n=4)$. Values were average means of triplicate experiments. Results were expressed as means \pm SEM. Statistical analysis used MannWhitney U-test. **P $<0.01$.

decreased mRNA expression levels of anti-inflammatory cytokines TGF- $\beta 1$ and IL-10 after LPS challenge in vitro. In accordance with our findings, Lcn2 was reported to induce IL-10 formation by macrophages and skew macrophage polarization. ${ }^{25} \mathrm{Lcn} 2$ has also been implicated in macrophage polarization by altering iNOS and Arg-1 levels after LPS challenge. ${ }^{26} \mathrm{MCP}-1$ is a potent chemokine of macrophages, and plays a critical role in inflammatory healing in extravascular tissues such as cutaneous wounds and myocardial infarction via a MIP-2 dependent pathway. ${ }^{27}$ In the present study, the mRNA expression of chemokines MCP-1 and MIP-2 were both lower in $\mathrm{Lcn} 2^{-/-}$macrophages, which suggested that Lcn2 deficiency would decelerate the migration of cells and tissue healing.

Macrophages are central participants in sterile and infectious chronic inflammatory syndromes. To define the mechanism by which Lcn 2 regulates LPS-challenged macrophage inflammation, a label-free proteomics analysis was performed. Similar to the results in mice, the levels of pro-inflammatory factor-related proteins were increased significantly, while those of anti-inflammatory factor-related proteins were decreased in
LPS-treated BMDMs of $\mathrm{Lcn}^{-/-}$mice compared with those of WT mice. Phagocytosis capacity of macrophage also plays an important role in cellular immunity, inflammation and tissue homeostasis. Effective suppression of inflammation is critical not only for the termination of the inflammatory response, but also for the restoration of tissue integrity. ${ }^{28}$ Phagocytosis of dead cells by macrophages not only plays an important role in clearance of apoptotic cells, but also promotes a pro-lytic phenotype of macrophages, thus terminating inflammation. ${ }^{29}$ Correspondingly, our results exhibited dampened phagocytosis of BMDMs from $\mathrm{Lcn} 2^{-/}$mice and aggravated inflammatory damage in $\mathrm{Lcn} 2^{-/-}$mice. The clearance of apoptotic cells by macrophages is coordinated by recognizing the primary "eatme" signal, which is present outside the membrane of dying cells. ${ }^{30}$ Mfge8, a bridging molecule that mediates binding of these signals to phagocytic receptors, effectively promotes phagocytosis of macrophage. ${ }^{31}$ Our study found that there was a decreased protein expression level of Mfge8 during LPSinduced inflammation in BMDMs of $\mathrm{Lcn} 2^{-/}$mice, whose impaired phagocytosis were approved by flow cytometric analyses. 


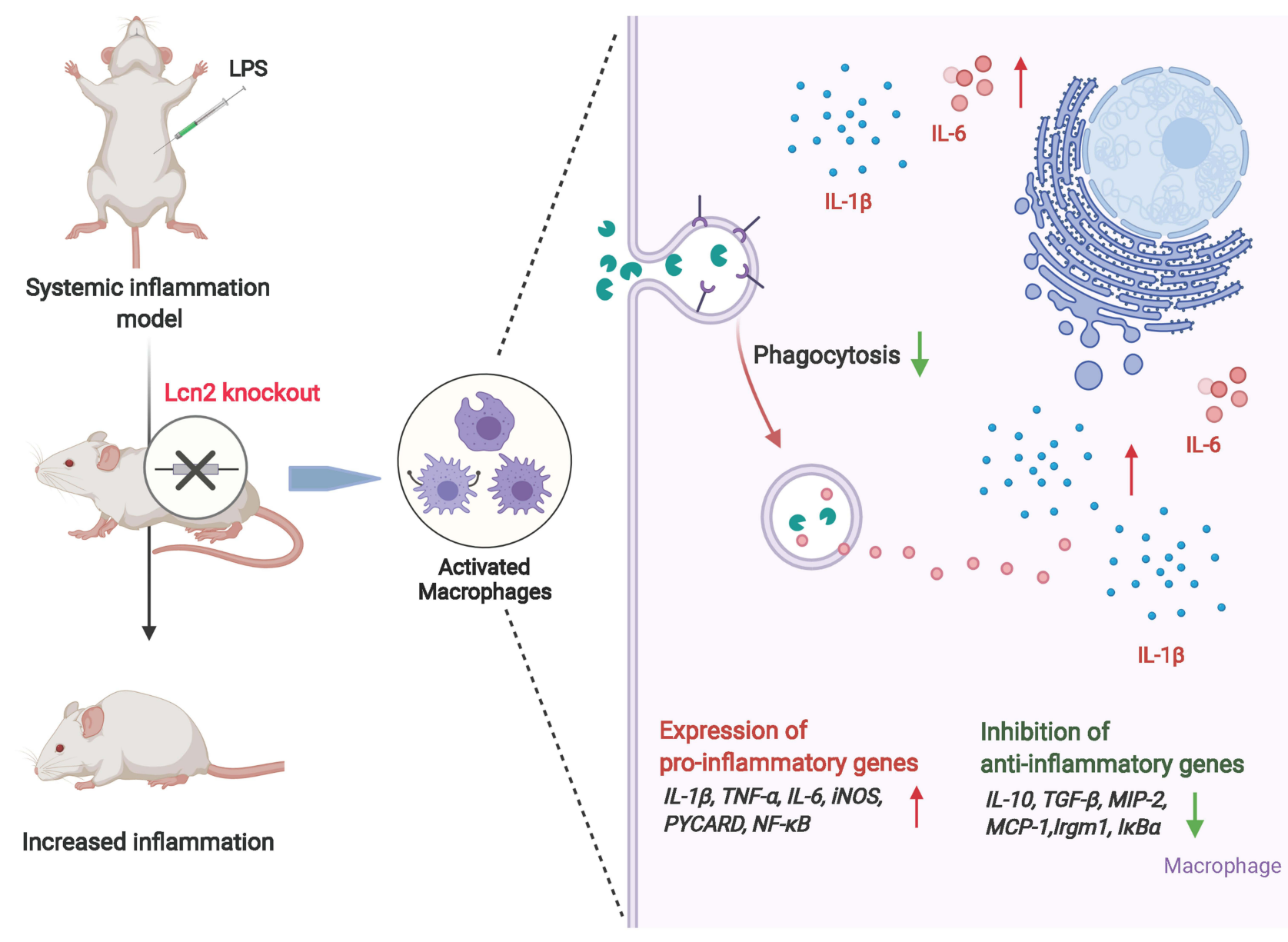

Figure 8 The mechanism of the pro-inflammatory effect of Lcn2 knockout in LPS-induced inflammation. The schemata was created with BioRender.com.

Macrophages phagocytize a wide range of exogenous particles such as necrotic cells arising from infection and inflammation, accompanied by features in common with autophagy. ${ }^{32}$ Our proteomics data also showed that autophagy related proteins such as PYCARD and Irgm1 were altered significantly in LPS-challenged BMDMs between WT and Lcn $2^{-/}$genotypes. Autophagy, as a fundamental cell survival process, plays a broad homeostatic role in the clearance of cytotoxic wastes, and has attracted much attention due to the importance in the protection from chronic inflammatory diseases. ${ }^{33}$ PYCARD complexes with NLRP3 protein to form a caspase-1-activating complex-NLRP3 inflammasome, which can cleave and activate IL-1 $\beta$ during inflammation. ${ }^{34} \operatorname{Irgm} 1$, one of the strongest cell autonomous resistance systems for autophagy of intracellular pathogens, ${ }^{35}$ also played an important role in negatively regulating IL- $1 \beta$ maturation by autophagy and degradation of PYCARD, thereby suppressing the activation of the NLRP3 inflammasome. ${ }^{33}$ In our study, we found and verified a significant reduction of
Irgm1 expression in BMDMs from $\mathrm{Lcn} 2^{-/}$mice, and correspondingly, a dramatic increase in PYCARD and IL-1 $\beta$, which finally resulted in reduced autophagy and increased inflammation.

In the present study, we provide evidence that LPS strongly induced Len2 that mainly localized to macrophages. Lcn2 deficiency could aggravate the inflammation with elevation of pro-inflammatory cytokines, and disturb the balance between pro-inflammatory and antiinflammatory responses of macrophages. Although proteomics analysis showed that LPS-treated BMDMs from $\mathrm{Lcn} 2^{-/-}$mice had increased levels of pro-inflammatory pathways, further studies will be needed to identify the exact function of these pathways. Moreover, we found that Lcn2 deficiency inhibited the phagocytosis of BMDMs. However, little is known if Lcn2 could affect the phagocytosis of macrophages in vivo, and further studies of the interaction between Lcn2 and autophagyrelated proteins are also needed to clarify the role of $\mathrm{Lcn} 2$ in inflammation. 


\section{Conclusion}

In conclusion, upon LPS-induced systemic inflammation, Lcn2 was produced and secreted in a parabolic pattern and mainly located in the cytoplasm of macrophages. Lcn2 deficiency can lead to enhanced pro-inflammatory response in both mice and macrophages challenged with LPS. It was due to not only the facilitating the expression of proinflammatory cytokines, but also the reduced autophagy of macrophages. Dampened phagocytosis and autophagy capacity of macrophages from $\mathrm{Lcn} 2^{-/-}$mice might also impede the resolution of inflammation (Figure 8). Our study suggests that Lcn2 is required to serve as a potent protective factor in response to systemic inflammation and highlights the importance of Lcn2 in keeping normal functions of macrophages.

\section{Abbreviations}

Len2, Lipocalin-2; LPS, lipopolysaccharide; WT, wildtype; BMDMs, bone marrow-derived macrophages; IL, interleukin; NF- $\mathrm{B}$, nuclear factor kappa-B; GO, Gene Ontology; KEGG, Kyoto Encyclopedia of Genes and Genomics; PYCARD, apoptosis-associated speck-like protein containing a CARD.

\section{Data Sharing Statement}

All data and materials supporting the conclusions were included in this paper. More details are available on request.

\section{Ethics Approval and Consent to Participate}

All procedures involving animals were approved and performed in accordance with the Animal Care and Use Committee of Zhejiang University.

\section{Acknowledgments}

We thank Shuhui Li for her great contribution to the experiments and we are very sorry about her passing away.

\section{Funding}

This work was supported by Natural Science Foundation of Zhejiang province of China (LZ20C170004, LY19HD30011), National Natural Science Foundation of China (31872363) and Major Science and Technology Project of Zhejiang province of China (2021C0306).

\section{Disclosure}

The authors report no conflicts of interest in this work.

\section{References}

1. Lin WW, Karin M. A cytokine-mediated link between innate immunity, inflammation, and cancer. J Clin Invest. 2007;117:1175-1183. doi: $10.1172 / \mathrm{JCI} 31537$

2. Kolb R, Sutterwala FS, Zhang W. Obesity and cancer: inflammation bridges the two. Curr Opin Pharmacol. 2016;29:77-89. doi:10.1016/ j.coph.2016.07.005

3. Hotamisligil GS. Inflammation and metabolic disorders. Nature. 2006;444:860-867. doi:10.1038/nature05485

4. Okin D, Medzhitov R. Evolution of inflammatory diseases. Curr Biol. 2012;22:R733-R740. doi:10.1016/j.cub.2012.07.029

5. Gordon S. Phagocytosis: an immunobiologic process. Immunity. 2016;44:463-475. doi:10.1016/j.immuni.2016.02.026

6. Epelman S, Lavine KJ, Randolph GJ. Origin and functions of tissue macrophages. Immunity. 2014;41:21-35. doi:10.1016/j. immuni.2014.06.013

7. Kjeldsen L, Johnsen AH, Sengeløv H, et al. Isolation and primary structure of NGAL, a novel protein associated with human neutrophil gelatinase. J Biol Chem. 1993;268:10425-10432. doi:10.1016/S00219258(18)82217-7

8. Flower DR, North AC, Sansom CE. The lipocalin protein family: structural and sequence overview. Biochim Biophys Acta. 2000;1482:9-24. doi:10.1016/S0167-4838(00)00148-5

9. Goetz DH, Holmes MA, Borregaard N, et al. The neutrophil lipocalin NGAL is a bacteriostatic agent that interferes with siderophore-mediated iron acquisition. Mol Cell. 2002;10:1033-1043. doi:10.1016/S1097-2765(02)00708-6

10. Devireddy LR, Gazin C, Zhu X, et al. A cell-surface receptor for lipocalin $24 \mathrm{p} 3$ selectively mediates apoptosis and iron uptake. Cell. 2005;123:1293-1305. doi:10.1016/j.cell.2005.10.027

11. Mishra J, Dent C, Tarabishi R, et al. Neutrophil gelatinase-associated lipocalin (NGAL) as a biomarker for acute renal injury after cardiac surgery. Lancet. 2005;365:1231-1238. doi:10.1016/S0140-6736(05) 74811-X

12. Liu Z, Petersen R, Devireddy L. Impaired neutrophil function in $24 \mathrm{p} 3$ null mice contributes to enhanced susceptibility to bacterial infections. J Immunol. 2013;190:4692-4706. doi:10.4049/jimmunol.1202411

13. Flo TH, Smith KD, Sato S, et al. Lipocalin 2 mediates an innate immune response to bacterial infection by sequestrating iron. Nature. 2004;432:917-921. doi:10.1038/nature03104

14. Singh V, Yeoh BS, Chassaing B, et al. Microbiota-inducible innate immune, siderophore binding protein lipocalin 2 is critical for intestinal homeostasis. Cell Mol Gastroenterol Hepatol. 2016;2:482-498. doi:10.1016/j.jcmgh.2016.03.007

15. Dittrich AM, Krokowski M, Meyer HA, et al. Lipocalin 2 protects against airway inflammation and hyperresponsiveness in a murine model of allergic airway disease. Clin Exp Allergy. 2010;40:1689-1700. doi:10.1111/j.1365-2222.2010.03508.x

16. Kang SS, Ren Y, Liu CC, et al. Lipocalin-2 protects the brain during inflammatory conditions. Mol Psychiatry. 2018;23:344-350. doi:10.1038/mp.2016.243

17. Nam Y, Kim JH, Seo M, et al. Lipocalin-2 protein deficiency ameliorates experimental autoimmune encephalomyelitis: the pathogenic role of lipocalin-2 in the central nervous system and peripheral lymphoid tissues. $J$ Biol Chem. 2014;289:16773-16789. doi:10.1074/jbc.M113.542282

18. Jang E, Lee S, Kim JH, et al. Secreted protein lipocalin-2 promotes microglial M1 polarization. FASEB J. 2013;27:1176-1190. doi:10.1096/fj.12-222257

19. Zhang J, Wu Y, Zhang Y, et al. The role of lipocalin 2 in the regulation of inflammation in adipocytes and macrophages. Mol Endocrinol. 2008;22:1416-1426. doi:10.1210/me.2007-0420

20. Moschen AR, Adolph TE, Gerner RR, et al. Lipocalin-2: a master mediator of intestinal and metabolic inflammation. Trends Endocrinol Metab. 2017;28:388-397. doi:10.1016/j.tem.2017.01.003 
21. Wang Q, Li S, Tang X, et al. Lipocalin 2 protects against Escherichia coli infection by modulating neutrophil and macrophage function. Front Immunol. 2019;10:2594. doi:10.3389/fimmu.2019.02594

22. Tang W, Ma J, Gu R, et al. Lipocalin 2 suppressed ocular inflammation by inhibiting the activation of NF- $\mathrm{B}$ pathway in endotoxininduced uveitis. Cell Physiol Biochem. 2018;46:375-388. doi:10.1159/000488472

23. Shi C, Pamer EG. Monocyte recruitment during infection and inflammation. Nat Rev Immunol. 2011;11:762-774. doi:10.1038/ nri3070

24. Ginhoux F, Jung S. Monocytes and macrophages: developmental pathways and tissue homeostasis. Nat Rev Immunol. 2014;14:392-404. doi:10.1038/nri3671

25. Warszawska JM, Gawish R, Sharif O, et al. Lipocalin 2 deactivates macrophages and worsens pneumococcal pneumonia outcomes. J Clin Invest. 2013;123:3363-3372. doi:10.1172/JCI67911

26. Guo H, Jin D, Chen X. Lipocalin 2 is a regulator of macrophage polarization and NF- $\mathrm{KB} / \mathrm{STAT} 3$ pathway activation. Mol Endocrinol. 2014;28:1616-1628. doi:10.1210/me.2014-1092

27. Hoh BL, Hosaka K, Downes DP, et al. Monocyte chemotactic protein-1 promotes inflammatory vascular repair of murine carotid aneurysms via a macrophage inflammatory protein-1 $\alpha$ and macrophage inflammatory protein-2-dependent pathway. Circulation. 2011;124:2243-2252. doi:10.1161/CIRCULATIONAHA.111.036061
28. Han CZ, Juncadella IJ, Kinchen JM, et al. Macrophages redirect phagocytosis by non-professional phagocytes and influence inflammation. Nature. 2016;539:570-574. doi:10.1038/nature20141

29. Kourtzelis I, Hajishengallis G, Chavakis T. Phagocytosis of apoptotic cells in resolution of inflammation. Front Immunol. 2020;11:553. doi:10.3389/fimmu.2020.00553

30. Ravichandran KS. Find-me and eat-me signals in apoptotic cell clearance: progress and conundrums. $J$ Exp Med. 2010;207:1807-1817. doi:10.1084/jem.20101157

31. Hanayama R, Tanaka M, Miwa K, et al. Identification of a factor that links apoptotic cells to phagocytes. Nature. 2002;417:182-187. doi:10.1038/417182a

32. Levine B, Mizushima N, Virgin HW. Autophagy in immunity and inflammation. Nature. 2011;469:323-335. doi:10.1038/nature09782

33. Deretic V, Klionsky DJ. Autophagy and inflammation: a special review issue. Autophagy. 2018;14:179-180. doi:10.1080/ 15548627.2017.1412229

34. Schroder K, Kanneganti TD, Shao F, et al. Mechanisms and consequences of inflammasome activation. J Mol Biol. 2018;430:131-132. doi:10.1016/j.jmb.2017.12.005

35. Singh SB, Davis AS, Taylor GA, et al. Human IRGM induces autophagy to eliminate intracellular mycobacteria. Science. 2006;313:1438-1441. doi:10.1126/science.1129577
Journal of Inflammation Research

\section{Publish your work in this journal}

The Journal of Inflammation Research is an international, peerreviewed open-access journal that welcomes laboratory and clinical findings on the molecular basis, cell biology and pharmacology of inflammation including original research, reviews, symposium reports, hypothesis formation and commentaries on: acute/chronic inflammation; mediators of inflammation; cellular processes; molecular

\section{Dovepress}

mechanisms; pharmacology and novel anti-inflammatory drugs; clinical conditions involving inflammation. The manuscript management system is completely online and includes a very quick and fair peerreview system. Visit http://www.dovepress.com/testimonials.php to read real quotes from published authors. 